\title{
Zu F. v. Winckels 70. Geburtstage.
}

Von M. Hofmeier.

Am 5. Juni dieses Jahres feiert Franz v. Winckel seinen 70. Geburtstag. Der Aufforderung der Redaktion dieser Wochenschrift, ihr einen Begrüßungsartikel für den Jubilar zu diesem festlichen Tage zu liefern, bin ich um so lieber gefolgt, als das Schicksal es gefügt hat, daß ich, abgesehen von persönlichen Beziehungen, seit einer langen Reihe von Jahren an der zwreitgrößten bayerischen Landesuniversität tätig, vielfach Gelegenheit hatte, das Wirken des hochgeschätzten Kollegen und Jubilars zu verfolgen, auch wo es nicht so sehr an die Oeffentlichkeit getreten ist. „Unser Leben währet $70 \mathrm{Jahre}$, und wenn es hoch kommt, sind es $80 \mathrm{Jahre}$ und wenn es köstlich gewesen ist, so ist es Mühe und Arbeit gewesen." Dieses Wort des Psalmisten könnte der Jubilar wohl als Motto iiber seinen Lebenslauf setzen, wenn er am 5. Juni rückblickend die sieben Jahrzehnte seines Lebens im Geiste überschaut. Denn in der Tat: ein Leben, reich an Arbeit, reich an Erfolgen liegt hinter ihm, wie es den wenigsten Sterblichen vergönnt ist, zu leben. Nur in kurzen Zügen sei der äußere Lebensgang von v. Winckel hier skizziert.

Franzv. Winckel wurde als Sohn des in der geburtshilflichen Literatur, besonders als erfolgreicher Kaiserschnittoperateur, aufs beste bekannten Kreisarztes und Sanitätsrates L u dwig Winckel in Berleburg in Westphalen geboren. Ich erinnere mich noch lebhaft daran, diesen verehrungsund bewunderungswürdigen Mann mit seinem weißen Haupt auf einer Naturforscherversammlung in früheren Jahren gesehen zu haben, diesen Mann, der noch im 80. Lebensjahr imstande war, seine Praxis auszuüben und unter äußerst ungünstigen Verhältnissen auf dem Lande einen Kaiserschnitt mit Erfolg ausżuführen. ${ }^{\text {) }}$ Ihm konnte noch die große Freude zuteil werden, an seinem 60 jährigen Doktorjubiläum ein bedeutendes Werk aus der Feder

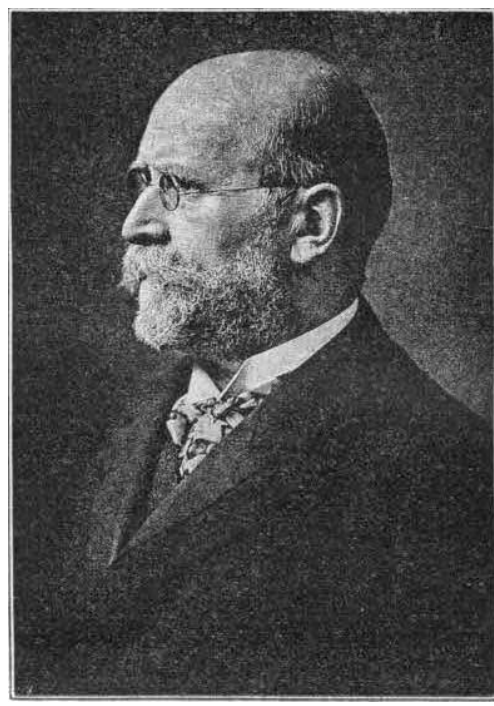
seines Sohnes zu empfangen.

Auch der Großvater W inckel war schon Arzt und Geburtshelfer gewesen, und somit war das Interesse für die Medizin, und insbesondere für die Geburtshilfe, dem Sohn und Enkel eigentlich erblich überkommen. Dieser widmete sich denn auch nach Absolvierung des Gymnasiums gleich dem Studium der Medizin und trat, wie sein Vater, in Berlin als Zögling in das medizinisch-chirurgische Friedrich WilhelmsInstitut ein, aus welchem ja eine so große Reihe später so hervorragender Aerzte hervorgegangen ist. Als Schüler von Schoeller und E. Martin promovierte v. Winckel im Jahre 1860 mit einer Dissertation: De partu praematuro arte efficiendo und trat nach Vollendung seiner medizinischen. Studien 1861 als Assistent unter E. Martins Leitung in die Universitäts-Frauenklinik ein. Als Sekundärarzt dieser Anstalt erhielt v. Winckel bereits im 29. Lebensjahr die Berufung als ordentlicher Professor der Geburtshilfe und Gynäkologie an die Universität Rostock.

In dieser Stellung verblieb er bis 1872, wo er einer Berufung an die Königl. Hebammenlehranstalt in Dresden folgte, welche unter seiner Leitung (bis 1883) sich allmählich zu der Bedeutung entwickelte, die sie unter den Schwesteranstalten in den deutschen Landen heute einnimmt. Im Jahre 1883 folgte v. Winckel dann als Nachfolger von Hecker dem Rufe an die Universität München, deren Lehrstuhl er ja noch heute ziert.

Wenn auch r. Winckel während seiner Dresdener Jahre eine akademische Lehrtätigkeit im engeren Sinne nicht ausgeübt hat, so hat er doch auch in dieser. Zeit nicht nur ununterbrochen an dem wissenschaftlichen Leben lebhaftesten Anteil genommen, sondern durch den Unterricht der zahlreichen, internen Hilfsärzto an dem Dresdener Institut auch andauernd eine Lehrtätigkeit zur Ausbildung von Aerzten ausgeübt, sodaß er an seinem Jubiläumstage auf eine 43 jährige, der Ausbildung von Aerzten gewidmete Lehrtätigkeit zurückblicken kann. Ungezählte Generationen von Aerzten verdanken ihm also ihre geburtshilfliche und gynäkologische Ausbildung, Aerzte aus allen Weltteilen und Weltgegenden, und dankbar werden sie am 5. Juni ihres Lehrers und Meisters gedenken.

1) Zentralblatt für Gynăkologie 1889. 
Es kann nicht meine Aufgabe sein, an dieser Stelle und bei dieser Gelegenheit eine eingehende Würdigung der in diesem langen Zeitraum geleisteten wissenschaftlichen Arbeit v. Winckels zu geben. Schier unübersehbar ist die Zahl der von ihm selbst ausgegangenen Veröffentlichungen, literarischen Leistungen, Vorträge in gelehrten Gesellschaften etc. Kaum ein Gebiet in unserem Fache, insbesondere in der Geburtshilfe, gibt es, das nicht von ihm neue, befruchtende Anregung erhalten hätte, oder in welchem er nicht durch die Mitteilung seiner reichen Erfahrung fördernd gewirkt hätte. Nur um einige der Hauptwerke v. Winckels zu nennen, seien hier angeführt seine Monographie „Ueber Pathologie und Therapie des Wochenbettes, Berlin 1866“, „Die Krankheiten der weiblichen Harnröhre und Blase“ in Billroths Handbuch der Frauenkrankheiten 1877, „Die Pathologie der weiblichen Sexualorgane in Lichtdruckabbildungen“, Leipzig 1881, „Berichte und Studien aus dem Königl. Entbindungsinstitut zu Dresden“, 3. Bd., „Lehrbuch der Frauenkrankheiten“, Leipzig 1886 und das „Lehrbuch der Geburtshilfe ${ }^{*}$ 1889. Außerdem leitete v. Winckel die Wiederausgabe des vortrefflichen Buches von Ammon „Die ersten Mutterpflichten", gab mit Credé zusammen das sächsische Hebammenlehrbuch heraus und leitete zusammen mit Bergmann und Erb, später mit Hildebrand und Müller seit Volkmanns Tode die Herausgabe der berïhmten „Klinischen Vorträge“. Trotz der außerordentlichen Arbeitslast, welche aus seiner klinischen, praktischen und akademischen Tätigkeit, sowie aus seiner amtlichen Stellung im Obermedizinalausschuß etc. und aus der tätigen Mitgliedschaft so vieler gelehrter Gesellschaften sich für ihn ergibt, haben uns die letzten Jahre immer wieder neue und sehr wertvolle literarische Gaben aus seiner Feder gebracht, von denen ich nur nennen möchte: „Ueber die Mißbil. dungen ektopisch entwickelter Früchte", Wiesbaden 1902, "Ueber die Entstehung, Einteilung und Benennung der Bildungshemmungen der weiblichen Sexualorgane", Klinische Vorträg૯. N. F. No. 251, und den ganz kürzlich erst erschienenen, geistvollen Aufsatz über die Gynäkologie Shakespeares, in welchem sich uns v. Winckel als feiner Kenner der klassischen Literatur zeigt. Dasjenige Werk aber, welches am meisten mit dazu beitragen wird, v. Winckels Namen für immer in der Geschichte der Geburtshilfe zu erhalten, ist das von ihm redigierte und seinem Abschluß sich jetzt nähernde, große Handbuch der Geburtshilfe: ein Standardwork der deutschen Literatur, dem sich so leicht kein ähnliches an die Seite stellt. v. Winckel selbst hat in diesem Buche außer einer Reihe von einzelnen, mit gewohnter Gründlichkeit und umfassender Sachkenntnis geschriebenen Abschnitten vor allem die historische Einleitung geschrieben, in welcher wir von neuem wieder seine umfassende Literaturkenntnis und sein tiefes Literaturstudium bewundern können. Auf eine sachliche Würdigung des wissenschaftlichen Inhaltes dieser Werke kann hier nicht eingegangen werden. Nur das mag gesagt sein, daß sie ausnahmslos das Gepräge des v. Winckelschen Geistes tragen: absolute Exaktheit, sorgfältiges Studium, objektivste kritische Sichtung. In allen seinen Arbeiten ist $v$. Winckel dem Worte treu gefolgt, welches er dem ersten Band seiner Berichte und Studien aus dem Dresdener Institut voransetzte: "Im Leben bekommt man nichts umsonst. Wer gehört sein und sein Wort geachtet wissen will, muß sich um die Solidität seines Urteils bemühen und darf die strenge Vorarbeit nicht meiden."

Wenn in dem Vorstehenden versucht ist, in ganz kurzen Zügen einen Umriß von v. Winckels eminenter literarischer und wissenschaftlicher Arbeit zu geben, so soll darüber nicht vergessen sein, mit welchem Interesse und von welchem hohen Standpunkt aus v. W inckel allezeit seine akademische Lehraufgabe aufgefaßt hat. Der in den „Berichten und Studien aus der Königlichen UniversitätsFrauenklinik in München", Leipzig 1892, S. 409 abgedruckte klinische Vortrag v. Winckels: "Ueber die klinische und praktische Ausbildung des Arztes" legt hierfür ein beredtes Zeugnis ab.

v. Winckel kann geradezu als ein Organisator des akademischen Unterrichts bezeichnet werden, und viele seiner Schüler werden den leichten Zwang, der mit seiner Methode des Unterrichts verbunden ist, gewiß später dankbar anerkannt haben.

Dieses große Organisationstalent hat auch v. Winckel in der Förderung und Ordnung der ihm unterstellten Institute in Dresden sowohl wie in München glänzend bewiesen. Es ist ja bekannt, und v. Winckel hat es wiederholt öffentlich ausgesprochen und in Privatgesprächen geschildert, unter welchen eminent ungünstigen und widrigen Verhăltnissen er die Klinik in München übernehmen und zunächst leiten mußte. Nur wer solche Verhältnisse kennt und zu würdigen weiß, wird ermessen können, welch große Fortschritte das Institut seiner Leitung in jeder Beziehung verdankt.

Die größte Förderung erfuhr dasselbe durch die im Jahre 1900 stattfindende Eröffnung des Neubaues der Hebammenschule, welcher, auf einem sehr ungtinstigen Terrain angelegt, doch in einer mustergültigen Weise den Bedingungen entspricht, die an ein solches In- stitut zu stellen sind. Hierdurch wurde die lange ersehnte "und jedenfalls sehr wünschenswerte, vollkommene räumliche Trennung des Hebammenunterrichts von dem akademischen Unterricht ermöglicht. Es spricht sich auch in ihr das hohe Interesse aus, welches v. Winckel allezeit dem Hebammenwesen und der Förderung des Hebammenunterrichts entgegengebracht hat. Dasselbe Organisationstalent hat $v$. W inckel bewiesen, indem er im Jahre 1885 in München die Gynäkologische Gesellschaft und im Jahre 1884 in Straßburg zusammen mit anderen die Deutsche Gesellschaft für Geburtshilfe und Gynäkologie begründete, welche sich dann zum ersten Male 1886 unter seinem Präsidium in München versammelte.

Daß ein Mann von so universeller naturwissenschaftlicher Bildung und organisatorischem Talent auch außerhalb des engeren Kreises seiner Fachgenossen sich des höchsten Ansehens erfreut, bewies die hohe Auszeichnung, daß man ihn zweimal in München und Meran zum ersten Vorsitzenden der Deutschen Naturforschergesellschaft wählte. Alle, welche diese Gesellschaften besucht haben, werden sich noch mit Vergnügen der glänzenden Leitung derselben durch ihren Vorsitzenden erinnern.

Von der reichen Arbeit, welche diese ausgedehnte und mannigfache Tätigkeit der verflossenen Jahrzehnte ihm brachte, suchte v. Winckel Erholung und Zerstreuung auf zahlreichen Reisen im In- und Ausland, indem er sie zugleich benutzte, seine Welt- und Menschenkenntnis zu erweitern, alte freundschaftliche, persönliche Beziehungen zu erneuern, neue anzuknüpfen. Doch dienten die seit vielen Jahren regelmäßig im Frühling in Pegli, im Herbst in Sylt genommenen, längeren Ferienaufenthalte nicht allein der Erholung, sondern wesentlich auch zur ruhigen, nicht durch die Tagestätigkeit gestörten Arbeit.

Daß das an äußerem Erfolg und Ehren so reiche Leben des Jubilars auch durch ein glückliches Familienleben beglückt und verschönt wurde, wissen alle, die das Glück hatten, einmal in diesem Familienkreise mit ihm zu verkehren. Mit aufrichtigem Mitgefühl haben wir alle an dem schweren und unersetzlichen Verlust teilgenommen, der dieses schöne Familienglück vor kurzem durch den Tod eines von allen, die ihn kannten, so hoch geschätzten Kollegen und Mitgliedes der Familie v. Winckel so schwer betroffen hat.

Daß es dem Jubilar auch an äußeren Ehrungen und Anerkennungen nicht gefehlt hat, ist bekannt; wir betrachten sie nur als den dem Verdienste gebührenden, äußerlichen Tribut. Eine wesentlich größere Befriedigung wird $v$. Winckel an seinem 70. Geburtstag jedenfalls darin finden, daß sich in dankbarer Beglückwünschung an diesem Tage zahllose seiner früheren Schüler und Schülerinnen ihres Lehrers und Meisters erinnern, daß $\mathrm{ihm}$ in neidloser Anerkennung seiner hervorragenden Persönlichkeit seine Fachkollegen ihre Huldigung an diesem Festtage darbringen, daß ungezählte Frauen, welche seiner ärztlichen Kunst Leben und Gesundheit verdanken, in herzlichster Dankbarkeit ihm ihre Gedanken und Glückwünsche zuwenden. So möge es der Jubilar auch uns gestatten, zu seinem Festtage, neben dem Ausdruck persönlicher Verehrung und Dankbarkeit, auch im Namen dieser Wochenschrift und ihres weiten Leserkreises ihm die herzlichsten Gläckwünsche und unsere aufrichtige Huldigung darzubringen. Möge ein gütiges Geschick dem verehrten Manne und stets so liebenswürdigen Kollegen noch lange die körperliche und geistige Frische erhalten, in welcher wir alle ihn seit so vielen Jahren kennen! Ad multos annos! 